CONTIPELLI, Ernani. Soberanía, Gobernanza Global y Mega-Regionalismos: Perspectivas Latinoamericanas. Revista Eletrônica Direito e Política, Programa de Pós-Graduação Stricto Sensu em Ciência Jurídica da UNIVALI, Itajaí, v.12, n.3, 30 quadrimestre de 2017. Disponível em: www.univali.br/direitoepolitica - ISSN 1980-7791

\title{
SOBERANÍA, GOBERNANZA GLOBAL Y MEGA-REGIONALISMOS: PERSPECTIVAS LATINOAMERICANAS
}

\author{
SOVEREIGNTY, GLOBAL GOVERNANCE AND MEGA-REGIONALISMS: \\ LATIN AMERICAN PERSPECTIVES
}

\section{Ernani Contipelli ${ }^{1}$}

ÍNDICE: Introducción; 1 Soberanía y Gobernanza Global; 2 Mega-regionalismos; 3 Perspectivas Latinoamericanas; Consideraciones Finales; Referencia de las fuentes citadas

\section{RESUMEN}

El presente articulo tiene como finalidad discutir la relación entre soberanía y gobernanza global, señalando la importancia de los procesos de integración regional, en especial, con los denominados mega-regionalismos desde la perspectiva Latinoamericana. Así, en la primera parte, investigamos la influencia del proceso de globalización en la esfera de soberanía de los Estados-nación y la consecuente necesidad de institucionalización de un sistema de gobernanza global. Posteriormente, ingresamos en el estudios de los mega-regionalismos, analizando su concepto y las principales iniciativas impulsadas por China y los EEUU para la implementación de tal modelo de gobernanza. Por fin, constatamos las perspectivas latinoamericanas de formación de un mega-bloque regional a partir del proceso de transformación político-ideológico del Mercosur y de creación de la Alianza Pacífica, así como las posibles negociaciones realizadas por ambos para formación de una grande coalición regional.

PALABRAS-CLAVES: Gobernanza Global; Mega-regionalismos; Mercosur; Alianza Pacífico

\begin{abstract}
The present article aims to discuss the relation between sovereignty and global governance, highlighting the importance of the process of regional integration, especially, with the mega-regionalism from the perspective of Latin America. Therefore, in the first part, we will investigate the influence of the globalization

\footnotetext{
1 Pos-Doctor en Derecho Político Comparado - Universidad Pompeu Fabra (España). Pos-Doctor en Derecho Constitucional Comparado - Universidad Complutense de Madrid (España). Doctor en Derecho del Estado - PUC/SP (Brasil). Máster en Filosofía del Derecho y del Estado - PUC/SP (Brasil). Máster en Derecho Tributario - PUC/SP (Brasil). Licenciado en Derecho - Mackenzie/SP (Brasil). Profesor del Programa de Posgrado en Derecho de la Universidade Comunitária da Região de Chapecó (Brasil). Director del Center for European Strategic Research (Italia). Email: ernanicontipelli@gmail.com.
} 
CONTIPELLI, Ernani. Soberanía, Gobernanza Global y Mega-Regionalismos: Perspectivas Latinoamericanas. Revista Eletrônica Direito e Política, Programa de Pós-Graduação Stricto Sensu em Ciência Jurídica da UNIVALI, Itajaí, v.12, n.3, $3^{\circ}$ quadrimestre de 2017. Disponível em: www.univali.br/direitoepolitica - ISSN 1980-7791

process in the sphere of sovereignty of the Nation-State and the necessity of institutionalization of a system of global governance. Sequentially, we will study the mega-regionalism, analyzing its concept and the main initiatives promoted by China and USA in order to implement it. At the end, we will verify the Latin American perspectives of composition of mega-regional institution from the political- ideological transformation of Mercosur and the creation of the Pacific Alliance, as well as the possible negotiation driven by both in order to form a large regional coalition.

KEYWORDS: Global Governance; Mega-regionalism; Mercosur; Pacific Alliance

\section{INTRODUCCIÓN}

Los paradigmas determinantes de la construcción del Estado-nación se presentan debilitados ante el cambio de las sociedad contemporánea determinado por la intensificación de la globalización, que exige las institucionalización de mecanismos que posibiliten un adecuado enfrentamiento de los denominados "problemas sin pasaporte".

Ante tal realidad, el orden internacional presupone un progresivo aumento de las relaciones de interdependencia entre Estados-nación a favor de la institucionalización de un futuro sistema de gobernanza global, momento en que debemos observar las transformaciones experimentadas por los procesos de integración, especialmente, con los denominados mega-regionalismo y sus perspectivas en distintas regiones del planeta.

El presente articulo tiene como finalidad discutir la relación entre soberanía y gobernanza global, señalando la importancia de los procesos de integración regional, en especial, con los denominados mega-regionalismos desde la perspectiva Latinoamericana.

Así, en la primera parte, investigamos la influencia del proceso de globalización en la esfera de soberanía de los Estados-nación y la consecuente necesidad de institucionalización de un sistema de gobernanza global. Posteriormente, ingresamos en el estudios de los mega-regionalismos, analizando su concepto y las principales iniciativas impulsadas por China y los EEUU para la implementación de tal modelo de gobernanza. 
CONTIPELLI, Ernani. Soberanía, Gobernanza Global y Mega-Regionalismos: Perspectivas Latinoamericanas. Revista Eletrônica Direito e Política, Programa de Pós-Graduação Stricto Sensu em Ciência Jurídica da UNIVALI, Itajaí, v.12, n.3, 30 quadrimestre de 2017. Disponível em: www.univali.br/direitoepolitica - ISSN 1980-7791

Por fin, constatamos las perspectivas latinoamericanas de formación de un mega-bloque regional a partir del proceso de transformación político-ideológico del Mercosur y de creación de la Alianza Pacífica, así como las posibles negociaciones realizadas por ambos para formación de una grande coalición regional.

\section{SOBERANÍA Y GOBERNANZA GLOBAL}

El aumento de la interdependencia entre naciones motivada por la intensificación del proceso de globalización despierta la atención del mundo para los denominados riesgos globales, es decir, los problemas comunes de nuestro planeta como medio ambiente, paz, desarrollo humano, pobreza, entre otros, que, por su emergencia, una vez que son directamente vinculados a la propia sobrevivencia de la especie humana y tienden a convertirse en la prioridad de las agendas políticas internacionales y nacionales, exigiendo una respuesta conjunta, un esfuerzo cooperativo y solidario entre las naciones para afrontarlos con la búsqueda de soluciones adecuadas.

Ante los riesgos globales, es evidente la debilidad del Estado-nación y de su modelo de soberanía absoluta como estructura de poder apropiada para el trato de las señaladas cuestiones, lo que demuestra la necesidad de generación de nuevas formas institucionales de cooperación internacional y que caminen hacia la instauración de una gobernanza global².

Por lo tanto, es cierto que el mundo sufre profundas transformaciones que generaran el surgimiento de problemas enfrentados en escala global, incrementando así el nivel de interdependencia en los países que pasan a buscar

\footnotetext{
2 La gobernanza consiste en un sistema de normas orientadas a la coordinación y colaboración entre distintos actores, para repartir los costes y beneficios de acciones conjuntas. En el ámbito de las relaciones internacionales, la gobernanza es comprendida a partir de dos perspectivas: como procesos institucionales que establecen la cooperación entre diferentes actores en la esfera internacional, suprimiendo la ausencia de un "Estado Mundial"; o manifestación compartida de poder en la esfera internacional, de tal modo que tal papel esta encargado a los Estados, así como a demás actores no-estatales. Desde de tales consideraciones iniciales, comprendemos que la gobernanza trata de reducir el impacto de los aspecto negativos del proceso de globalización y, por otro lado, establecer incentivos y extender la cooperación internacional, estimulando la realización de acciones destinadas a la concreción de los aspectos positivos del señalado proceso (ANDREATTA, F., CLEMENTI, M., COLOMBO, A., KOENIG-ARCHIBUGI, M., PARSI, V. E. Relazioni Internazionali. Bologna: Mulino, 2007).
} 
CONTIPELLI, Ernani. Soberanía, Gobernanza Global y Mega-Regionalismos: Perspectivas Latinoamericanas. Revista Eletrônica Direito e Política, Programa de Pós-Graduação Stricto Sensu em Ciência Jurídica da UNIVALI, Itajaí, v.12, n.3, $3^{\circ}$ quadrimestre de 2017. Disponível em: www.univali.br/direitoepolitica - ISSN 1980-7791

nuevos modelos de alianzas e integración para lograr una efectiva y solidaria cooperación en el ámbito internacional, los cuales afectan la confortable condición de "only international player" de los Estados-nación y por consecuencia de la propia noción de soberanía ${ }^{3}$.

En tal sentido, David Held señala que en la actualidad se reconoce que los problemas globales no pueden ser resueltos por un Estado-nación actuando aisladamente, ni tampoco por Estados que sólo luchan por ocupar un lugar en sus bloques regionales.

Así, el autor pondera que en la medida que han ido aumentando las exigencias al Estado, ha ido surgiendo un conjunto de problemas políticos que no pueden ser adecuadamente sin la cooperación de otros Estados y de actores no estatales, de modo que cada vez se tiene más conciencia de que los Estados ya no son las únicas unidades políticas apropiadas, ya sea para resolver los principales problemas políticos, ya para gestionar la amplia gama de funciones publicas ${ }^{4}$.

En definitivo, la condición actual de la humanidad impone la búsqueda por formulas de organización social que posibiliten un ajuste entre el poder institucional y los efectos de la globalización, que consoliden la idea de interdependencia y solidaridad entre naciones para enfrentar problemas comunes y de orden planetaria, lo que exige la flexibilización del paradigma de la soberanía absoluta propia de la figura Estado-nación y una mayor interacción política en el ámbito supranacional, como forma de establecer un apropiado camino para lograr una futura gobernanza global.

De ese modo, la gobernanza global surge como meta a ser lograda por la humanidad en su intento de desarrollar una conciencia solidaria en nivel planetario, para promover una mejor cooperación entre las naciones y contener los nocivos efectos de los riesgos globales.

3 CONTIPELLI, E. Estado Constitucional Cooperativo: Perspectivas sobre Solidaridad, Desarrollo Humano y Gobernanza Global. Revista Inciso vol. 18, p. 84, 2016.

${ }^{4}$ HELD, D. Cosmopolitismo, Ideales y Realidades, p. 37, 2010. 
CONTIPELLI, Ernani. Soberanía, Gobernanza Global y Mega-Regionalismos: Perspectivas Latinoamericanas. Revista Eletrônica Direito e Política, Programa de Pós-Graduação Stricto Sensu em Ciência Jurídica da UNIVALI, Itajaí, v.12, n.3, 30 quadrimestre de 2017. Disponível em: www.univali.br/direitoepolitica - ISSN 1980-7791

Lógicamente, la gobernanza global es un largo camino, una proyección para el futuro que orienta nuestra percepción del presente, que nos guía en el análisis de la cooperación internacional y sus rumbos actuales, constatando las medidas que están siendo adoptadas para su debida instauración, especialmente, en términos de generación de estructuras de poder regionales e inter-regionales que posibiliten un acercamiento de intereses comunes entre naciones.

En otras palabras, los procesos de integración desempeñan un papel decisivo en la consagración de un futura estructura de gobernanza global, en la medida que una gobernanza región permite la persecución de objetivos externos, domésticos y transnacionales convergentes en determinada zona geográfica delimitada a través de estructuras de poder reconocidas institucionalmente, estrechando eventuales lazos de cooperación y posibilitando una actuación dinamizada a favor del trato de los múltiples intereses que no pueden ser solucionados aisladamente por los Estados-naciones.

Por lo tanto, los procesos de integración regional deben ser visualizados históricamente como una etapa anterior a una futura y necesaria implementación de un modelo de gobernanza global. En otras palabras, los bloques de integración regional funcionan como un elemento institucional intermedio que posibilita la apertura de un canal de comunicación entre los Estados-nación y la prospectiva de formación de un "Estado Mundial"5. Estamos conscientes de que el camino es largo, caracterizado por una serie de obstáculos y dificultades, sin embargo, el momento histórico en que vivimos orientase a favor del

\footnotetext{
${ }^{5}$ Interesante apuntar la idea de Estado Mundial desarrollada por Alexander Wendt que presupone la existencia de una entidad de carácter supranacional que atendiera la necesidad de establecer una estructura coercitiva que posibilite la integración global de las naciones respetando sus diversidades en un ambiente de paz y seguridad. Conforme el pensamiento del autor, contemporáneamente, competiría a la Organización de las Naciones Unidas convertirse en tal instancia de decisión jerárquicamente superior en la esfera internacional, ejerciendo las atribuciones de un "Estado Mundial" a través de un modelo de gobernanza inclusiva que permitiera las manifestaciones democráticas de múltiples campos de interés: económico, cultural, científico, entre otros, reduciendo así eventuales conflictos entre diferentes percepciones de mundo. Un punto a ser destacado en las consideraciones de Wendt respecta a la Unión Europea, la cual debe ser comprendida como una especie de prototipo en términos de gobernanza comunitaria supranacional, por su compleja estructura institucional que busca homogenizar intereses en un espacio históricamente caracterizado por divergencias a través de la adopción de una política, al menos normativamente, de contenido consensual y democrático que visa la solución de problemas comunes de la región. (WENDT, A. Why a World State is Inevitable, en European Journal of International Relations, 9 (4), 491-542, 2003).
} 
CONTIPELLI, Ernani. Soberanía, Gobernanza Global y Mega-Regionalismos: Perspectivas Latinoamericanas. Revista Eletrônica Direito e Política, Programa de Pós-Graduação Stricto Sensu em Ciência Jurídica da UNIVALI, Itajaí, v.12, n.3, $3^{\circ}$ quadrimestre de 2017. Disponível em: www.univali.br/direitoepolitica - ISSN 1980-7791

regionalismo y de sus múltiples y reciprocas relaciones, para promover un acercamiento entre las naciones y ciudadanos $\mathrm{y}$, principalmente, afrontar sus problemas comunes.

Considerando, entonces, la institucionalización de una futura gobernanza global como premisa de investigación, podemos verificar el comportamiento de los actores internacionales y sus practicas políticas en el mundo contemporáneo y como ellas están enfrentando y buscando soluciones para los riesgos globales, por intermedio de estructuras de gobernanza regional, como el caso de los mega-regionalismos.

\section{MEGA-REGIONALISMOS}

En tal contexto, encontramos los Mega-regionalismos, también denominados de "Mega Bloques", los cuales, considerados por Barack Obama como "marco comercial del siglo XXI", consisten, básicamente, en modelos de cooperación internacional con características especificas por involucrar países política y económicamente estratégicos en el escenario mundial (por sus referencias en términos de población, producto, comercio, inversión extranjera, afinidad política, etc..), para creación de espacios económicos con amplia repercusión geográfica al conectar mercados en diferentes regiones y con complejas agendas que superan las temáticas tratadas por simples acuerdos de libre comercio entre naciones, al establecer marcos regulatorios convergentes sobre factores directa y indirectamente vinculados a materias comerciales, como, por ejemplo, regulaciones ambientales y laborales.

Las afirmaciones anteriores pueden perfectamente comprobadas en los datos relativos al Acuerdo de Asociación Económica Regional (Regional Comprehensive Economic Partnership, RCEP), que actualmente se encuentra en proceso de negociación y consistirá en el mayor acuerdo de libre comercio del mundo, por incluir los participantes de la $\operatorname{ASEAN}+6^{6}$, los cuales representan 3.000 millones

${ }^{6}$ El Acuerdo de Asociación Económica Integral Regional (RCEP, sigla en ingles) que involucra los países miembros de la ASEAN (Asociación de Naciones del Sudeste Asiático, compuesta por Indonesia, Malasia, Filipinas, Singapur, Tailandia, Brunei, Myanmar, Camboya, Laos, Vietnam) y las naciones con las cuales poseen tratados de libre comercio en vigencia (Australia, China, India, Japón, Corea del Sur y Nueva Zelandia). 
CONTIPELLI, Ernani. Soberanía, Gobernanza Global y Mega-Regionalismos: Perspectivas Latinoamericanas. Revista Eletrônica Direito e Política, Programa de Pós-Graduação Stricto Sensu em Ciência Jurídica da UNIVALI, Itajaí, v.12, n.3, 30 quadrimestre de 2017. Disponível em: www.univali.br/direitoepolitica - ISSN 1980-7791

de personas, un PIB de 17.000 millones de dólares y un total de $40 \%$ del comercio mundial ${ }^{7}$, involucrando los siguientes sectores: el comercio de bienes y servicios, la inversión, la cooperación económica y técnica, la propiedad intelectual, la competitividad y la resolución de disputas.

Otro ejemplo que puede ser señalado es el Acuerdo de Asociación Transpacífico (o TPP, sigla en ingles), que, ya consumado en el 05 de octubre de 2015, prevé la eliminación de barreras arancelarias y no arancelarias entre 12 países pertenecientes a la región Asia-Pacífico (Estados Unidos, Japón, Australia, Brunei Canadá, Chile, Malasia, México, Nueva Zelandia, Perú, Singapur y Vietnam), representando $11 \%$ de la población, $38 \%$ del PIB y $23 \%$ de las exportaciones mundiales.

Además del TPP, otro importante mega acuerdo, que, actualmente, está en proceso de negoción: el Acuerdo Transatlántico de Comercio e Inversión entre Unión Europea y Estados Unidos (TTIP, sigla en ingles), que abarcaría el 45\% del PIB y el $40 \%$ de las exportaciones mundiales; y, englobando, así, el $48 \%$ de la población, 30\% del PIB y de las exportaciones mundiales.

No obstante, la intensa negociación realizada por la administración Obama, que determino la firma del TPP y ha estimulado el desarrollo de iniciativas como el TTIP, la llegada de Donald Trump a la Presidencia de los Estados Unidos y su propuesta de cambiamiento de la política exterior practicada por su predecesor, determino la retirada del país del acuerdo y paralizó demás negociaciones, implicando concretamente en un retroceso en la conclusión de acuerdos megaregionales, por la perdida de interés por parte de los demás signatarios en desarrollarles sin la presencia de su gran protagonista, los EEUU ${ }^{8}$.

\footnotetext{
7 MURRAY, H., LIAM H. ASEAN and Partners Launch Regional Comprehensive Economic Partnership (en linea), Center for strategic \& international studies, Strategic insights and bipartisan policy solutions. Disponible: http://csis.org/publication/asean-and-partners-launchregional-comprehensive-economic-partnership, 2012.

8 Con el éxito de Donald Trump en las elecciones presidenciales de 2016 y su propuesta proteccionista de "American First", una de sus primeras e impactantes medidas están relacionadas con la retirada de los EEUU del TPP, eliminando la estrategia de política exterior planteada por Barak Obama. Entre las consecuencias del acto de Trump, señalamos las incertidumbres con relación a la continuidad de participación de importantes miembros en el TPP, como Japón y
} 
CONTIPELLI, Ernani. Soberanía, Gobernanza Global y Mega-Regionalismos: Perspectivas Latinoamericanas. Revista Eletrônica Direito e Política, Programa de Pós-Graduação Stricto Sensu em Ciência Jurídica da UNIVALI, Itajaí, v.12, n.3, 30 quadrimestre de 2017. Disponível em: www.univali.br/direitoepolitica - ISSN 1980-7791

Por su parte, China apoya firmemente el RCEP para consolidar su papel ante el actual orden mundial. Interesante que algunos miembros de la ASEAN+6 (Brunei, Singapur, Vietnam, Malasia, Japón, Australia y Nueva Zelandia) estaban negociando su participación en ambos acuerdos, RCEP y TPP, estimulando la competencia entre China y los EEUU. La opción de algunos países asiáticos de unirse a los dos acuerdos demuestra como a través de un modelo de política exterior esencialmente pragmática buscan maximizar los beneficios que las grandes potencias pueden proporcionar en sus respectivas regiones.

Interesante apuntar que el RCEP se caracteriza por un sistema de funcionamiento basado en el ASEAN way, en que sus objetivos y compromisos son determinados a partir del consenso y del respeto a los diferentes niveles de desarrollo económico de sus miembros, mientras el TPP tiene la ambición de fijar otro nivel de compromiso, con mayor vinculación entre los países miembros. El "ASEAN way" puede ser comprendido como un sistema de principios que forman la base de funcionamiento del regionalismo en Asia Oriental, los cuales favorecen la discusión para lograr el consenso, y no un carácter típicamente legalista, propio del sistema occidental ${ }^{9}$.

Aunque los acuerdos mega-regionales pretendan establecer un modelo de referencia para la reorganización de la estructura comercial global, evidentemente, que ellos encuentran fuerte resistencia por amplios sectores de la sociedad, en razón de una serie de factores que van desde las negociones secretas para conformación de los acuerdos hasta el temor por los impactos que pueden ser generados en términos de aumento de las tasas de paro y desconstrucción de la empresa nacional, por fuerza de la gran competitividad y

Canadá. Además surgen una serie de especulaciones sobre la configuración de una "post-nuevo orden mundial", que camina para el aislamiento de los EEUU al multilateralismo y, simultáneamente, la apertura de espacios políticos para que China consolide una posible leadership en el ámbito internacional.

\footnotetext{
${ }^{9}$ De acuerdo con Amitav Acharya (Constructing a Security Community in Southeast Asia: ASEAN and the Problem of Regional Order. New York : Routledge, p. 26, 2001), el "ASEAN way" se caracteriza por cuatro principios básicos: la ausencia de formalidades, la no injerencia, la consulta y la búsqueda del consenso (consensus-building).
} 
CONTIPELLI, Ernani. Soberanía, Gobernanza Global y Mega-Regionalismos: Perspectivas Latinoamericanas. Revista Eletrônica Direito e Política, Programa de Pós-Graduação Stricto Sensu em Ciência Jurídica da UNIVALI, Itajaí, v.12, n.3, 30 quadrimestre de 2017. Disponível em: www.univali.br/direitoepolitica - ISSN 1980-7791

concesión de subsidios por las grandes potencias a sectores estratégicos de la economía, como la agricultura.

Entre las cuestiones más discutidas y contestadas, principalmente, con respecto al TTIP, señalamos la clausula del Investor-State Dispute Settlement (ISDS), que permite a los inversores extranjeros promover un procedimiento de arbitraje para solución de diferencias contra el Estado-nación anfitrión, exigiéndole significativas indemnizaciones por modificaciones políticas que puedan afectar las condiciones comerciales iniciales ${ }^{10}$. Ante la controversia generada por la inclusión de la ISDS en las propuestas de conclusión del TTIP, en 2014, la Comisión Europea promovió una consulta publica, que demostró el amplio rechazo de los participantes ( $97 \%$ de oposición) en relación a tal clausula.

Desde la perspectiva latinoamericana, nos interesa el análisis del actual momento político vivenciado por la región y como se refleja en términos de cooperación internacional y la posible contribución de un modelo de gobernanza regional que genere condiciones para la institución de una futura gobernanza global, lo que nos lleva a discutir las relaciones entre la Alianza Pacífico y el Mercosur.

\section{PERSPECTIVAS LATINOAMERICANAS}

Antes de ingresar en la situación actual de los bloques anteriormente señalados, necesitamos regresar en el tiempo para mejor comprender el proceso de construcción del regionalismo sudamericano a partir del Mercosur.

El Mercosur fue creado en la década de los 90 del siglo pasado, a partir da firma del Tratado de Asunción entre Argentina, Brasil, Paraguay y Uruguay, como forma de actualizar las políticas de desarrollo de sus participantes, orientándolas a la liberalización comercial, reducción de barreras arancelarias e integración al mercado internacional.

10 Conforme los datos de la UNCTAD, en 2015, los procedimientos arbitrales iniciados por inversores extranjeros utilizando el modelo "Investor-State Dispute Settlement" ha alcanzado 696 demandas, en que la alegación fue basada en el argumento argumento de que los Estados-nación anfitrión no estarían respetando los compromisos asumidos en los acuerdos internacionales de inversión (World Investment Report 2016: Investor Nationality Policy Challenges). 
CONTIPELLI, Ernani. Soberanía, Gobernanza Global y Mega-Regionalismos: Perspectivas Latinoamericanas. Revista Eletrônica Direito e Política, Programa de Pós-Graduação Stricto Sensu em Ciência Jurídica da UNIVALI, Itajaí, v.12, n.3, $3^{\circ}$ quadrimestre de 2017. Disponível em: www.univali.br/direitoepolitica - ISSN 1980-7791

Con respecto al contexto en que se forma el MERCOSUR, Álvaro Vasconcelos comenta que el bloque puede ser considera el primero proyecto de integración generado por la globalización, a partir de una actitud consciente de Brasil y Argentina sobre las dificultades enfrentadas por las naciones en desarrollo para superar los desafíos económicos durante tal momento histórico ${ }^{11}$.

Así, el MERCOSUR surge como una respuesta a las necesidades de sus miembros de cambiar las políticas de desarrollo implementadas en las décadas de 60 y 70 direccionadas a la industrialización y consecuente sustitución de las importaciones para actualízalas y oriéntalas a la apertura comercial e integración en el mercado internacional, es decir, ajustadas a las propuestas neo-liberales del Washington Consensus.

En siglo XXI, motivado por el cambio de orientación político-ideológico en el continente sudamericano, constatamos una ampliación de los objetivos del Mercosur, para imponerle una connotación de carácter social en su estructura institucional, especialmente, con la iniciativa de creación de los siguientes órganos:

a) Instituto Social del MERCOSUR, aprobado en la Reunión de Ministros y Autoridades de Desarrollo Social y creado por Decisión del Consejo Mercado Común n. 03/2007, tiene como propósito la investigación en el campo de las políticas sociales, para promover y generar acciones de carácter inclusivo, con equidad e igualdad;

b) Instituto de Políticas Públicas en Derechos Humanos (IPPDH), creado por Decisión del Consejo del Mercado Común n. 14/09, está orientado a la investigación, capacitación y apoyo en la coordinación de políticas regionales de los derechos humanos, entre las cuales se destacan las políticas de memoria, verdad, justicia y reparación ante graves violaciones; políticas de igualdad y no

\footnotetext{
11 VASCONCELOS, A European Union and MERCOSUR, p. 166/167 en TELO, M. (ed.) European Union and New Regionalism: Regional Actors and Global Governance in a Post-Hegemonic Era, Hampshire: Ashgate, 2007.
} 
CONTIPELLI, Ernani. Soberanía, Gobernanza Global y Mega-Regionalismos: Perspectivas Latinoamericanas. Revista Eletrônica Direito e Política, Programa de Pós-Graduação Stricto Sensu em Ciência Jurídica da UNIVALI, Itajaí, v.12, n.3, 30 quadrimestre de 2017. Disponível em: www.univali.br/direitoepolitica - ISSN 1980-7791

discriminación; políticas de prevención de la violencia institucional y seguridad ciudadana e infraestructura en derechos humanos; y, por fin,

c) PARLASUR (o Parlamento del MERCOSUR), aprobado por el Programa de Trabajo 2004-2006 del Consejo del Mercado Común, se constituye en 2006, como espacio político de representación del pluralismo y de las diversidades contenidas en el territorio del bloque, para cumplir funciones testimoniales en defesa de la democracia y de los derechos humanos ${ }^{12}$.

Además, en ese periodo, considerando los vínculos político-ideológicos entre los gobiernos de las naciones interesadas y la apertura de las directrices establecidas por el Tratado de Asunción, con respecto a la aceptación de otros Estados miembros de Asociación Latinoamericana de Integración, el MERCOSUR pasa a incorporar dos nuevos países, Venezuela y Bolivia.

Venezuela firma el protocolo de adhesión al MERCOSUR en 2006, sin embargo, tal proceso apenas se completa en 2012, tras la suspensión de Paraguay en las decisiones del bloque, en razón de sus problemas domésticos, relacionados con la destitución del entonces presidente Fernando Lugo y la ausencia de elecciones democráticas en el país. Así, a partir de 30 de julio de 2012, con Paraguay alejado de sus funciones ante el MERCOSUR, los miembros restantes aprobaron el ingreso de Venezuela como miembro pleno, atribuyendo mayor peso político a la entidad, una vez que pasa a contar con una población de cerca de 275 millones de personas, equivalentes al 70\% del total de Sudamérica y un Producto Interno Bruto (PIB) de 3,32 billones de dólares, esto es, el 83 por ciento del de la región, además de una extensión territorial correspondiente a $72 \%$ de toda región (12,7 millones de kilómetros cuadrados).

En el caso de Bolivia, el país firmo el Protocolo de Adhesión al MERCOSUR en 2012, pero solamente en 2015 la totalidad de los Estados miembros del bloque reconoció tal documento, que, actualmente, aguarda su incorporación en los

\footnotetext{
12 Sobre el PARLASUR importante esclarecer que, aunque se caracterice como órgano político, no posee facultades decisorias, con actuación restricta a la elaboración de ciertos dictámenes no vinculantes, padeciendo, así, de los mismos problemas de legitimidad y competencias de los demás parlamento regionales latinoamericanos.
} 
CONTIPELLI, Ernani. Soberanía, Gobernanza Global y Mega-Regionalismos: Perspectivas Latinoamericanas. Revista Eletrônica Direito e Política, Programa de Pós-Graduação Stricto Sensu em Ciência Jurídica da UNIVALI, Itajaí, v.12, n.3, 30 quadrimestre de 2017. Disponível em: www.univali.br/direitoepolitica - ISSN 1980-7791

respectivos congresos nacionales. Mientras no se completa el referido tramite legal, Bolivia es considerada como "miembro en proceso de adhesión" con derecho a voz, pero sin voto en las decisiones del bloque.

Sin embargo, en la primera década del siglo XXI, constatamos un nuevo giro ideológico en Sudamérica, acreditado por los emblemáticos resultados obtenidos por proyectos políticos de derecha en la región, como la victoria del candidato Mauricio Macri en las elecciones presidenciales en Argentina, colocando fin al peronismo de los Kirchner; el debilitamiento del chavismo en Venezuela, con el éxito de la oposición en las elecciones legislativas; la derrota de Evo Morales en el referéndum destinado a aprobar su cuarta reelección en el gobierno del país; y, finalmente, el impeachment de Dilma Roussef, que cierra los 16 años de dominación del escenario político del país por el Partido de los Trabajadores, con el retorno de un gobierno de centro-derecha.

Otro factor que contribuye para el cambio ideológico del Mercosur está relacionado con el fin del superciclo económico experimentado a partir del boom de las commodities y la desaceleración del crecimiento de China, lo que lleva al interés de los países miembros de bloque por la flexibilización de las reglas negóciales del MERCOSUR, principalmente, en lo que se refiere a la posibilidad de establecimiento de acuerdos comerciales de manera unilateral ${ }^{13}$.

Paralelamente, a los revueltos políticos del Mercosur, en la mismo región, especialmente, en los Países ubicados en la zona del Pacífico, fue desarrollada una propuesta de política exterior direccionada al libre comercio, inicialmente, con la participación bilateral en acuerdos comerciales firmados con grades potencias, EEUU y Unión Europea, siendo que tal movimiento gana fuerza al punto de posibilitar, en 2011, la creación de una nueva entidad de cooperación

\footnotetext{
13 Con la firma del Protocolo de Ouro Preto en 1994, fue establecido el Arancel Externo Común, determinando la unión aduanera y las reglas de relaciones comerciales entre los miembros del bloque, mediante la cual se comprometen a "negociar en forma conjunta acuerdos de naturaleza comercial con terceros países o grupo de países extrazonas en los cuales se otorguen preferencias comerciales", conforme Decisión del Consejo del Mercado Común n. 32/00, impidiendo la negoción unilateral de nuevas preferencias comerciales, lo que, por un lado, permitió una mayor cohesión entre el grupo, pero, por otro, dificulto la implementación de un código aduanero común para el desarrollo de las propuestas de integración, convirtiendo la región en un zona de libre comercio.
} 
CONTIPELLI, Ernani. Soberanía, Gobernanza Global y Mega-Regionalismos: Perspectivas Latinoamericanas. Revista Eletrônica Direito e Política, Programa de Pós-Graduação Stricto Sensu em Ciência Jurídica da UNIVALI, Itajaí, v.12, n.3, 30 quadrimestre de 2017. Disponível em: www.univali.br/direitoepolitica - ISSN 1980-7791

internacional, la Alianza Pacifico, compuesta por Chile Perú, Colombia y México, la cual presenta objetivos básicos muy bien definidos para promoción de la inserción internacional de tales países: apertura economía y condiciones favorables para inversión extranjera, de manera que sus miembros son libre para establecer acuerdos comerciales unilateralmente ${ }^{14}$.

La idea contenida en tales objetivos es expresa en el Acuerdo Marco de la AP, suscrito en Antofagasta (Chile) en 06 de junio de 2012, que determina en su articulo 30: la construcción de "una área de integración profunda para avanzar progresivamente hacia la libre circulación de bienes, servicios, capitales y personas"; el impulso a "un mayor crecimiento, desarrollo y competitividad" de las economías de los países miembros, buscando "lograr un mayor bienestar, la superación de la desigualdad socioeconómica y la inclusión social de sus habitantes"; y, por fin, la conversión del bloque en "una plataforma de articulación política, de integración económica y comercial y de proyección al mundo, con especial énfasis en Asia-Pacífico".

Con respecto a la cuestión de los objetivos de la AP, Jairo Andrés Castaño Peña comenta que:

El propósito de la Alianza es lograr la libre circulación de bienes, servicios, capital y personas dentro de la región. Por otra parte, una de las prioridades del proceso de integración es lograr una consolidación que favorezca el intercambio comercial con otros mercados, especialmente con los países de la cuenca del Pacífico asiático. Es notorio que la atención de la Alianza está centrada en la profundización de las relaciones económicas con un corte aperturista, dejando de lado el componente político y social que ha caracterizado a otras iniciativas de integración latinoamericanas ${ }^{15}$.

\footnotetext{
${ }^{14}$ Importante resaltar el juego geopolítico que se estableció en la región y sus piezas, pues los países miembros de la AP se presentan políticamente próximos a los EEUU, siendo considerados incluso sus aliados históricos en el continente, de modo que es nítido el apoyo prestado por la gran potencia a la formación del bloque, lo que demuestra una especie de respuesta al aumento de la influencia China en Sudamérica y también al modelo de regionalismo posliberal que se desarrollo durante la primera década del siglo XXI sostenido especialmente por la ola de gobiernos progresistas de izquierda que se institucionalizó en diversos naciones de la región.
}

15 CASTAÑO-PEÑA, J. A. Análisis y Perspectivas de la Alianza del Pacífico. Revista Estudios de Deusto, pp. 281-305, 2016. 
CONTIPELLI, Ernani. Soberanía, Gobernanza Global y Mega-Regionalismos: Perspectivas Latinoamericanas. Revista Eletrônica Direito e Política, Programa de Pós-Graduação Stricto Sensu em Ciência Jurídica da UNIVALI, Itajaí, v.12, n.3, $3^{\circ}$ quadrimestre de 2017. Disponível em: www.univali.br/direitoepolitica - ISSN 1980-7791

Podemos ejemplificar, nuevamente, el nítido perfil neoliberal demostrado por la AP en otra disposición de su Acuerdo Marco, la cual se refiere a la adhesión de nuevos miembros (art. 11), que, entre los requisitos establecidos, determina la necesidad de la nación postulante tener firmado un Acuerdo de Libre Comercio con uno de los países miembros, sujetándose a posterior aprobación por unanimidad del Consejo de Ministros del bloque. Ese es el caso de Costa Rica, que en febrero de 2014, a través de su Presidenta Laura Chinchilla, firmó una declaración para iniciar formalmente su proceso de incorporación en la AP, una vez que habría suscripto en 2013 un Acuerdo de Libre Comercio con Colombia, cumpliendo así con los requisitos fijados en el Acuerdo Marco.

Siguiendo la línea del raciocinio anterior, señalamos que la AP, por su pragmatismo y las disposiciones estructurales que dictan las relaciones entre sus miembros, basados en el modelo de regionalismo abierto, presenta un dinamismo funcional totalmente distinto de los demás procesos integracionistas contemporáneos de la región en aspectos claves como la vinculación directa de su agenda política a las posiciones e intereses de las naciones participantes, como en la UNASUR, o en la definición de sus objetivos que están orientados a la cooperación económico-comercial y no un sistema de relaciones para lograr un desarrollo integral, caso del ALBA ${ }^{16}$.

La ascensión de la Alianza Pacífico y su pragmática política comercial empieza a atraer nuevos interesados en se acercar al grupo, entre ellos, los principales socios del Mercosur, Brasil y Argentina, que ahora orientados a la adopción de practicas económicas que se ajustan perfectamente a las propuestas de liberalización defendidas por los demás miembros de la Alianza. Especialmente, en 2015, tras la crisis política que afecta Brasil y cierra el ciclo de poder del Partido de los Trabajadores, con la asunción del gobierno Michel Temer de nítida orientación ideológica liberal, así como en Argentina con la vigente presidencia de Mauricio Macri, ocurre un acercamiento entre el MERCOSUR y la AP, con la finalidad de establecer mecanismos de cooperación entre los dos bloques e

16 LeVÍ CORAL, M. y REgGiardo, G. La Alianza del Pacífico en el Regionalismo Sudamericano Actual. Revista Mexicana de Política Exterior, n. 106, pp. 187-204, 2016. 
CONTIPELLI, Ernani. Soberanía, Gobernanza Global y Mega-Regionalismos: Perspectivas Latinoamericanas. Revista Eletrônica Direito e Política, Programa de Pós-Graduação Stricto Sensu em Ciência Jurídica da UNIVALI, Itajaí, v.12, n.3, $3^{\circ}$ quadrimestre de 2017. Disponível em: www.univali.br/direitoepolitica - ISSN 1980-7791

intensificar diálogos para conformación de una amplia zona de libre comercial en la región, uniendo Pacífico y Atlántico ${ }^{17}$.

Actualmente, las articulaciones políticas han sido intensas en tal sentido, por ejemplo, juntamente con Uruguay y Paraguay, Argentina también fue admitida, en 2016, como socio observador de la Alianza Pacífico, siendo que tales países ya declararan oficialmente la intención de firmar un tratado de libre comercio con el bloque a través de un relanzamiento del Mercosur.

Por lo tanto, todo ese escenario suscita una serie de consecuencias que determinan el futuro del Mercosur y del propio proceso de integración en el continente sudamericano, como la flexibilización de las reglas comerciales del Mercosur, permitiendo que sus miembros realicen negociaciones comerciales unilateralmente, lo que implicaría en la conversión de la actual unión aduanera en una zona de libre comercio; o incluso el establecimiento de un acurdo entre Mercosur y Alianza Pacífico, con dimensiones mega-regionales y bajo el consenso de los miembros de ambos bloques, convergiendo los dos polos económicos de la región y posibilitando, así, una mayor libertad de negociación entre los países involucrados, con la eventual formación de amplia zona de libre comercio en la región.

\section{CONSIDERACIONES FINALES}

Considerando la situación anteriormente presentada desde las perspectivas latinoamericanas. Por un lado, un acuerdo con dimensiones mega-regionales entre Mercosur y Alianza Pacífico demostraría un estrechamiento de los lazos de cooperación entre las regiones del continente y un primero paso en términos de consagración efectiva de una gobernanza regional, aunque por la vía de conformación de una amplia zona de libre comercio, una vez que las demás proyecciones para el Mercosur, en razón de su fragmentación interna, implicaría en un nítido retroceso en sus propuestas integracionistas.

\footnotetext{
17 PAIKIN y PERROTTA. La Argentina y la Alianza Pacífico: Riesgos y Oportunidades de una Nueva Geopolítica. Revista Aportes para la Integracion Latinoamericana n. 34, pp. 67-101, 2016.
} 
CONTIPELLI, Ernani. Soberanía, Gobernanza Global y Mega-Regionalismos: Perspectivas Latinoamericanas. Revista Eletrônica Direito e Política, Programa de Pós-Graduação Stricto Sensu em Ciência Jurídica da UNIVALI, Itajaí, v.12, n.3, $3^{\circ}$ quadrimestre de 2017. Disponível em: www.univali.br/direitoepolitica - ISSN 1980-7791

Por otro lado, la ascensión de la Alianza Pacífico, con su seductora política de liberalización comercial y flexibilización de relaciones comerciales entre sus miembros, la cual se orienta en la construcción de un proyecto integracionista con preocupaciones estrictamente económico-comerciales, genera de cierto modo un agravante para afirmación de una entidad de carácter supranacional en la región, destinada al futuro establecimiento de una "gobernanza regional" y que contribuya para una eventual "gobernanza global" como representante del continente latinoamericano.

Ciertamente, para realizar afirmaciones precisas sobre el tema, es preciso estar atento a los próximos capítulos a respeto del proceso de integración en la región, pues un posible acuerdo general entre Alianza Pacífico y Mercosur, lo cual se dibuja con rasgos concretos ante la influencia del panorama político-ideológico actual, definiendo los rumbos del modelo de cooperación internacional a ser adoptado en la región, orientado a la priorización de intereses económicos y comerciales.

\section{REFERENCIA DE LAS FUENTES CITADAS}

ACHARYA, A. Constructing a Security Community in Southeast Asia: ASEAN and the Problem of Regional Order. New York : Routledge, 2001.

ANDREATTA, F., ClEMENTI, M., COLOMBO, A., KOENIG-ARCHIBUGI, M., PARSI, V. E. Relazioni Internazionali. Bologna: Mulino, 2007.

CASTAÑO PEÑA, Jairo Andrés. Análisis y Perspectivas de la Alianza del Pacífico. Revista Estudios de Deusto, pp. 281-305. Bilbao: Universidad Deusto, 2016.

CONTIPELLI, E.. Estado Constitucional Cooperativo: Perspectivas sobre Solidaridad, Desarrollo Humano y Gobernanza Global. Revista Inciso vol. $18,2016$.

HELD, D. Cosmopolitismo: Ideales y Realidades. Madrid: Alianza Editorial, 2010. 
CONTIPELLI, Ernani. Soberanía, Gobernanza Global y Mega-Regionalismos: Perspectivas Latinoamericanas. Revista Eletrônica Direito e Política, Programa de Pós-Graduação Stricto Sensu em Ciência Jurídica da UNIVALI, Itajaí, v.12, n.3, $3^{\circ}$ quadrimestre de 2017. Disponível em: www.univali.br/direitoepolitica - ISSN 1980-7791

LEVÍ CORAL, Michel y REGGIARDO, Giulliana. La Alianza del Pacífico en el Regionalismo Sudamericano Actual. Revista Mexicana de Política Exterior, n. 106, pp. 187-204. México D.F.: Secretaria de Relaciones Exteriores, 2016.

MURRAY, H., LIAM H. ASEAN and Partners Launch Regiona Comprehensive Economic Partnership (en ligne), Center for strategic \& international studies, Strategic insights and bipartisan policy solutions. Disponible: http://csis.org/publication/asean-and-partners-launch-regional-comprehensiveeconomic-partnership, 2012.

PAIKIN, Damián y PERROTTA, Daniela. La Argentina y la Alianza Pacífico: Riesgos y Oportunidades de una Nueva Geopolítica. Revista Aportes para la Integracion Latinoamericana n. 34, pp. 67-101, 2016.

WENDT, A. Why a World State is Inevitable, en European Journal of International Relations, 9 (4), 491-542, 2003.

UNITED NATIONS CONFERENCE ON TRADE AND DEVELOPMENT. World Investment Report 2016: Investor Nationality Policy Challenges. Disponível em: <http://www.worldinvestmentreport.org> acceso en 30 de Octubre de 2017.

VASCONCELOS, A. European Union and MERCOSUR en TELO, M. (ed.) European Union and New Regionalism: Regional Actors and Global Governance in a Post-Hegemonic Era. Hampshire: Ashgate, 2007.

Submetido em: novembro de 2017

Aprovado em: dezembro de 2017 